\title{
Inverse Nonlinear Eigenvalue Problem Framework for the Synthesis of Coupled-Resonator Filters With Non-Resonant Nodes and Arbitrary Frequency-Variant Couplings
}

\author{
Martyna Mul, Adam Lamecki, Senior Member, IEEE, Roberto Gómez-García, Senior Member, IEEE, \\ and Michal Mrozowski, Fellow, IEEE
}

\begin{abstract}
A novel, general circuit-level description of coupledresonator microwave filters is introduced in this paper. Unlike well-established coupling-matrix models based on frequencyinvariant couplings or linear frequency-variant couplings (LFVCs), a model with arbitrary frequency-variant coupling (AFVC) coefficients is proposed. The engineered formulation is more general than prior-art ones and can be treated as an extension of previous synthesis models, since constant or linear couplings are special cases of arbitrary frequency dependence. The suggested model is fully general, allows for AFVCs with highly nonlinear (even singular) characteristics, loaded or unloaded nonresonating nodes (NRNs), frequency-dependent source-load coupling, multiple frequency-variant cross-couplings, and/or multiple dispersive couplings for connecting the source and load to the filter network. The model is accompanied by a powerful synthesis technique that is based on the zeros and poles of the admittance or scattering parameters and the eigenvalues of properly defined eigenproblems. In the most general case, the zeros and poles of the admittance or scattering parameters are related to solutions of nonlinear eigenvalue problems. The synthesis is defined as an inverse nonlinear eigenvalue problem (INEVP) where the matrix is constructed from three sets of eigenvalues. This is accomplished by optimization using an iterative nonlinear least-squares solver with excellent convergence property. Finally, third- and fifthorder examples of bandpass filter topologies involving AFVCs are shown, and the experimental validation of the proposed theory is presented through the manufacturing and characterization of a microstrip filter prototype with transmission zeros (TZs).
\end{abstract}

Index Terms-Microwave filters, Coupling matrix, Synthesis.

\section{INTRODUCTION}

$\mathbf{C}$ OUPLED-resonator bandpass filters are one of the fundamental microwave components in RF front-end chains to pre-select the desired RF signals and suppress unwanted out-of-band interfering signals and noise. Whereas different design approaches to synthesize bandpass filters have become well established during the years, the coupling-matrix formulation has acquired a paramount importance among researchers since its inception [1]. This modeling framework is based on

M. Mul, A. Lamecki and M. Mrozowski are with the Faculty of Electronics, Telecommunications, and Informatics, Gdańsk University of Technology, 80-233 Gdansk, Poland (e-mail: martyna.mul@pg.edu.pl; adam.lamecki@ieee.org; m.mrozowski@ieee.org).

R. Gómez-García is with the Department of Signal Theory and Communications, Polytechnic School, University of Alcalá, 28871 Alcalá de Henares, Spain (e-mail: roberto.gomez.garcia@ieee.org).

This work was supported by the Polish National Science Centre under contract UMO-2019/33/B/ST7/00889. a lumped-element circuit representation of the filter, which reflects the topology of its resonator network and the strength and nature of the interactions between its resonating elements. Conventional techniques for coupling-matrix synthesis generally assume that the inter-resonator coupling structures, also referred to as impedance or admittance inverters, do not vary with frequency [2]-[5]. As a result, the accuracy of this model to fairly represent the overall response of the filtering device is mostly restricted to narrow-to-moderatebandwidth specifications. To partially circumvent this limitation by considering specific frequency-variation profiles for couplings, a new broadband model for the coupling matrix in the passband domain was introduced in [6] that results in coupling elements to be inversely proportional to frequency. In [7], a combination of frequency-dependent inductive or capacitive couplings was considered for higher modelling accuracy in wide-band spectral intervals. In both cases, the goal was to broaden the frequency range of the couplingmatrix representation of a filter and to be able to place the transmission zeros (TZs) at arbitrary positions, including a finite number of TZs at the zero and infinity frequencies. However, as already demonstrated during the first decade of this century, dispersion-i.e., frequency-dependence-in couplings can be conveniently exploited even for narrow-band bandpass filters in inline [8]-[11] or triplet [12] topologies to generate additional TZs without significantly increasing the complexity of the associated inter-resonator coupling arrangement.

Since the realization of high-selectivity bandpass filtering actions in compact circuits with simple inter-resonator coupling schemes is a desired feature, a new synthesis technique aimed at filtering networks with arbitrarily-connected dispersively-cross-coupled resonators was proposed in [13]. An advanced version of this synthesis method that includes resonant-type source-load coupling to produce $n+1$ TZs in $n$ th-order bandpass filters was subsequently suggested in [14], and then refined in [15] to also account for the resonatordetuning effect due to the non-ideal dispersive couplings. The three variants of this technique share, as common principle, the fact that the zeros and poles in a cross-coupled filter with linear frequency-variant coupling (LFVCs) or inverters (LFVIs) in the lowpass prototype domain are the roots of the characteristic equation for the generalized eigenvalue problem. On the other hand, new direct coupling-matrix synthesis techniques for 
inline and cascaded topologies with LFVCs have been lately developed, such as those in [16] and [17], respectively.

Although linear frequency variation in couplings is often sufficient to describe the filter behaviour accurately, couplings may exhibit more-complex frequency-dependent patterns, which can be employed to further increase selectivity. Examples of such generalized coupling networks for different RF technologies encompass coupling elements in the form of an iris with complex geometry [18] and an internal bypass metallic loop structure [19] in the case of waveguide filters, or even alternative types of source-load coupling with additional transmission-line and waveguide sections in the input/output accesses for planar and 3-D implementations. The latter case is especially suitable to further augment the number of produced TZs due to the transversal signal-interference phenomenon, so that increased attenuation levels can be achieved in multiples regions of the stopband range [20]-[23]. However, previouslymentioned coupling-matrix synthesis approaches are not fully applicable to such more-general filtering configurations.

In order to provide a systematic design methodology for a much-broader variety of filtering structures, a novel couplingmatrix model and synthesis technique which allows for arbitrary frequency variation of inverters are reported in this paper. Furthermore, since the inter-resonator couplings cannot be implemented as ideal inverters-i.e., two-port networks with admittance/impedance matrices having zero diagonal elements-while detuning the resonators at the same time, this loading effect is directly taken into account here during the synthesis process by additionally allowing for it to be frequency-dependent. Finally, in order to increase flexibility in terms of distinct classes of filter networks that can be synthesized by means of the devised filter synthesis procedure, the inclusion of non-resonating nodes (NRNs) is also considered. The frequency profile of AFVC can be highly nonlinear, and even show singularities that generate poles. To the best of the authors' knowledge, no coupling-matrix-based filter model and synthesis technique of such level of generality has been proposed in the technical literature to date.

The organization of the rest of the manuscript is as follows. In Section II, the theoretical foundations of the proposed coupling-matrix synthesis methodology formulated as an inverse eigenvalue problem are presented. In Section III, a new filter model is introduced. Next, the step-by-step synthesis procedure for filter networks with non-ideal arbitrary frequency-variant couplings (AFVCs) or inverters (AFVIs), which can also include NRNs to increase the variety of affordable filtering topologies, is described. Specific types of AFVCs particularized in transmission-line realizations are shown in Section IV, which are then used in Section $\mathrm{V}$ for several theoretical examples of equiripple-type third- and fifthorder bandpass filters with TZs derived from the engineered coupling-matrix synthesis method. In Section VI, the experimental results of a built proof-of-concept microstrip prototype associated to one of the third-order filter examples previously synthesized are provided. Finally, a summary and the main concluding remarks of this work are set out in Section VII.

\section{Synthesis of A COUPling Matrix AS AN INVERse EigenVAlue PROBlem}

\section{A. Theoretical Foundations}

The fundamental core of the proposed synthesis technique is the relationship existing between the zeros and poles of the short-circuit admittance or scattering parameters of the filter network and the eigenvalues of the coupling matrix and its two principal submatrices. Therefore, a few basic preliminaries related to various categories of eigenproblems will be given first. In the simplest case of a standard eigenvalue problem with one matrix $\mathbf{A}$, nontrivial solutions are sought of a matrix equation

$$
(\mathbf{A}-\lambda \mathbf{I}) \mathbf{x}=0
$$

where $\mathbf{I}$ is the identity matrix and $\lambda$ is a scalar that is called eigenvalue. This equation has nonzero solution(s) if the determinant of the matrix $(\mathbf{A}-\lambda \mathbf{I})$ is zero. Therefore, the eigenvalues are the roots of the characteristic equation $\operatorname{det}(\mathbf{A}-\lambda \mathbf{I})=0$. Analogously, the generalized eigenvalue problem with a pair of matrices $(\mathbf{A}, \mathbf{B})$ is defined as

$$
(\mathbf{A}-\lambda \mathbf{B}) \mathbf{x}=0
$$

so that the eigenvalues are the roots of

$$
\operatorname{det}(\mathbf{A}-\lambda \mathbf{B})=0 .
$$

This can be made even more general by considering a parameter-dependent matrix $\mathbf{A}(\lambda)$ and the nonzero solutions of equation $\mathbf{A}(\lambda) \mathbf{x}=0$. This equation defines a nonlinear matrix eigenvalue problem. Again, the eigenvalues are the roots of the characteristic equation $\operatorname{det} \mathbf{A}(\lambda)=0$. To find the eigenvalues for a given matrix, numerical procedures have to be applied. For standard and generalized eigenproblems, the QR and QZ algorithms are recommended [24]. For a nonlinear problem, the characteristic equation can be either solved directly by using a root finding algorithm [25][27] or indirectly, by applying a specialized technique for parameter-dependent matrices [28], [29].

After these preliminary remarks, the relation of various categories of eigenvalue problems to the synthesis of coupledresonator filters can be explained. To start with, let it be considered the conventional circuit description of a bandpass filter consisting of a two-port network. For such filter circuit, Kirchhoff's voltage law reads as follows:

$$
\mathbf{Z}_{n}(\omega) \mathbf{i}=\mathbf{e}
$$

where $\mathbf{Z}_{n}(\omega)$ is a frequency-dependent impedance matrix for the network, $\mathbf{i}$ is a vector of loop currents, and $\mathbf{e}$ is the voltage source vector. In a conventional coupled-resonator synthesis technique, Kirchhoff's equations are expressed in the lowpass prototype domain $\Omega$ as below:

$$
\Omega=\frac{1}{\Delta}\left(\frac{\omega}{\omega_{0}}-\frac{\omega_{0}}{\omega}\right)
$$

where $\omega_{0}$ is the center frequency of the bandpass filter and $\Delta$ is its fractional bandwidth. Using this frequency transformation the following formula is obtained for the entire network:

$$
\left[\mathbf{R}+j\left(\mathbf{M}_{c}+\Omega \mathbf{I}\right)\right] \mathbf{i}=\mathbf{e}
$$


where $\mathbf{M}_{\mathbf{c}}$ is the (symmetric) coupling matrix, $\mathbf{R}$ is the matrix corresponding to the source and load resistances (this matrix is zero except for the first and last element on the main diagonal that take the value of the internal resistance of the source and load, respectively), and $\mathbf{I}$ is the identity matrix. The reactive part of the network can be characterized by the short-circuit admittance parameters [5]. For instance, $y_{11}^{c}$ is given by

$$
y_{11}^{c}=-j\left[\mathbf{M}_{c}+\Omega \mathbf{I}\right]_{11}^{-1}=-j \frac{\operatorname{det}\left[\mathbf{M}_{\mathbf{c}}^{\prime}+\Omega \mathbf{I}^{\prime}\right]}{\operatorname{det}\left[\mathbf{M}_{\mathbf{c}}+\Omega \mathbf{I}\right]}
$$

where the prime symbol is used to denote the matrices with the first row and first column deleted. Such matrix will be called lower principal submatix. A similar expression can be formulated for the short-circuit output admittance $y_{22}^{c}$ except that an upper principal submatrix, obtained by deleting the last row and column of the matrix $\mathbf{M}_{\mathbf{c}}$, is used instead in the numerator. For the transadmittance parameter $y_{21}^{c}$, an analogous formula that involves a submatrix obtained from $\mathbf{M}_{\mathbf{c}}$ by deleting the first column and last row (upper right principal submatrix) can be derived. Referring to (3), it is then evident that the expression in the numerator and denominator for all admittance parameters is the characteristic equation for finding the eigenvalues. Hence, the poles of the admittance parameters are the eigenvalues of the coupling matrix $\mathbf{M}_{\mathbf{c}}$, while the zeros of the short-circuit admittance parameters are the eigenvalues of its appropriate principal submatrices. Equation (7) is valid for a traditional category of filters, in which the coupling elements are frequency-invariant. However, this assumption can be relaxed by allowing for linear frequency variation of the inverters (in the prototype domain) so that $\mathbf{M}_{\mathbf{c}}=\mathbf{M}_{\mathbf{0}}+\Omega \mathbf{M}_{\mathbf{1}}$. In such case, the input short-circuit admittance of the reactive part is as follows:

$$
y_{11}^{c}=-j\left[\mathbf{M}_{\mathbf{0}}+\Omega \mathbf{M}_{\mathbf{1}}\right]_{11}^{-1}=-j \frac{\operatorname{det}\left[\mathbf{M}_{\mathbf{0}}^{\prime}+\Omega \mathbf{M}_{\mathbf{1}}^{\prime}\right]}{\operatorname{det}\left[\mathbf{M}_{\mathbf{0}}+\Omega \mathbf{M}_{\mathbf{1}}\right]} .
$$

Again, the prime symbol is used to refer to the lower principal submatrix of a matrix. The expressions for the remaining shortcircuit admittance parameters have a similar form. As a result, the poles of the admittance parameters are eigenvalues of the symmetric matrix pencil $\left(\mathbf{M}_{\mathbf{0}}, \mathbf{M}_{\mathbf{1}}\right)$, while the zeros of the short-circuit admittance parameters are eigenvalues of pencils obtained from the pair $\left(\mathbf{M}_{\mathbf{0}}, \mathbf{M}_{\mathbf{1}}\right)$ by deleting an appropriate row and column. At this point, an observation can be made related to the equivalence of filters with constant couplings and filters with LFVCs. A symmetric generalized eigenvalue problem can be transformed to a standard one as follows:

$$
(\mathbf{A}-\lambda \mathbf{B}) \mathbf{x}=\left(\mathbf{B}^{1 / 2} \mathbf{A B}^{1 / 2}-\lambda \mathbf{I}\right) \mathbf{x}=0 .
$$

Obviously, the structure (nonzero pattern) of $\mathbf{B}^{1 / 2} \mathbf{A} \mathbf{B}^{1 / 2}$ is different from the structure of the pencil. From the filter synthesis perspective, this equivalently means that every filter network with LFVCs can be implemented as a filter with constant couplings, albeit with a different coupling scheme, as observed also in [8].

The matrix eigenvalue problems considered above arise from the expressions for the short-circuit admittance parameters derived from the KLC equations. These equations are quite general, so nothing prevents this study from going one step further and assume that the inverters in the coupling matrix exhibit arbitrary frequency-dependence. For this situation, all the elements of the reactance matrix will be frequencyvariant. As a result, the expression for the short-circuit input admittance of the reactive part adopts the following form:

$$
y_{11}^{c}=-j[\mathbf{X}(\Omega)]_{11}^{-1}=-j \frac{\operatorname{det}\left[\mathbf{X}^{\prime}(\Omega)\right]}{\operatorname{det}[\mathbf{X}(\Omega)]} .
$$

As in previous two cases, the formula in the numerator and denominator is the characteristic equation, this time for nonlinear eigenvalue problems involving the entire reactance matrix $\mathbf{X}(\Omega)$ and lower principal submatrix $\mathbf{X}^{\prime}(\Omega)$.

It is obvious that, regardless of the assumed frequency dependence of the reactance matrix, the zeros and poles of the admittance parameters are nothing else but the eigenvalues of properly defined matrices, or matrix pencils. Since the zeros and poles of the short-circuit admittance parameters for a given filter configuration can be derived analytically from the filtering function [5], the coupling matrix synthesis is in fact an inverse matrix eigenvalue problem. This problem, in linear algebra, refers to the construction of a matrix with the prescribed structure from its eigenvalues.

Based on the observations made so far, this section concludes with the definition of the coupling matrix synthesis in terms of inverse eigenvalue problem (IEVP). Note that depending on the frequency behaviour of the inverters, the IEVP may be of the standard, the generalized or, in the most general form, of the nonlinear type.

\section{Definition (Coupling Matrix Synthesis as an IEVP).}

Given the three known rational functions $y_{11}, y_{21}$, and $y_{22}$ corresponding to the desired short-circuit admittance parameters of a filter and the intended coupling routing scheme, the coupling matrix synthesis as an IEVP can be set as finding a symmetric reactance matrix $\mathbf{X}(\Omega)$ with the structure (i.e., locations of the nonzero elements) determined by the coupling routing scheme such that it satisfies the following conditions:

1) Its eigenvalues are the poles of $y_{11}$ and $y_{22}$.

2) The eigenvalues of its lower principal submatrix are the zeros of $y_{11}$.

3) The eigenvalues of its upper right principal submatrix are the zeros of $y_{21}$

4) The eigenvalues of its upper principal submatrix are the zeros of $y_{22}$.

Some additional remarks to be considered are given below.

Remark 1. To synthesise the reactive part of a lossless filtering network, only two rational functions are needed. However, for lossy filters, synthesis scenarios based on three sets have to be used [30].

Remark 2. For frequency-invariant inverters (FIIs) and dispersive inverters with linear frequency variation, the reactance matrix $\mathbf{X}(\Omega)$ is given by $\mathbf{M}_{\mathbf{c}}+\Omega \mathbf{I}$ and $\mathbf{M}_{\mathbf{0}}+\Omega \mathbf{M}_{\mathbf{1}}$, respectively, and the eigenvalues of $\mathbf{X}(\Omega)$ are simply the eigenvalues of the matrix $\mathbf{M}_{\mathbf{c}}$ or matrix pencil $\left(\mathbf{M}_{\mathbf{0}}, \mathbf{M}_{\mathbf{1}}\right)$,

Remark 3. For a large number of coupling schemes with frequency-invariant inverters, the solution to the IEVP involving the matrix $\mathbf{M}_{\mathbf{c}}$ can be found directly by a series of 
eigenvalue-preserving similarity transformations applied to the transversal matrix, which can be found analytically [5].

Remark 4. A solution of the IEVP for all three categories of frequency variation of inverters considered here can be formulated in terms of the optimization problem. The details will be presented in the next section.

Remark 5. In the IEVP framework, the synthesis problem can also be solved in the bandpass domain. In this case, only the nonlinear variant is applicable.

Remark 6. In contrast to standard and generalized eigenvalue problems, the number of eigenvalues in a nonlinear eigenvalue problem can be greater than the size of the matrix. This is especially true when the matrix elements are periodic-type functions. In this case, the synthesis gives rise to a network with a response that properly matches the filter specification only within a certain bandwidth.

\section{B. Alternative Formulations}

The formulation presented above based on short-circuit admittance parameters is not directly applicable to more-complex coupling schemes, such as those of filters involving multiple dispersive couplings for connecting the source and load as well as direct, possibly resonant-type, source-load connections. One possible alternative-more-general-formulation for the synthesis as an INEVP can be derived by considering the relationship that links the coupling matrix and the scattering parameters. For the frequency-invariant model, the following formula is obtained:

$$
S_{11}(\Omega)-1=2 j R_{s} \frac{\operatorname{det}\left(\mathbf{M}_{\mathbf{c}}^{\prime}-j \mathbf{R}^{\prime}+\Omega \mathbf{I}^{\prime}\right)}{\operatorname{det}\left(\mathbf{M}_{\mathbf{c}}-j \mathbf{R}+\Omega \mathbf{I}\right)} .
$$

In the above expression, it is immediate to recognise the characteristic equations for the eigenvalues of complex matrices $\mathbf{M}_{\mathbf{c}}^{\prime}-j \mathbf{R}^{\prime}$ and $\mathbf{M}_{c}-j \mathbf{R}$ for the numerator and denominator, respectively. Allowing for the inverters to exhibit an arbitrary frequency-variation profile, a generalised equivalent version of (11) can be written as follows:

$$
S_{11}(\Omega)-1=2 j R_{s} \frac{\operatorname{det}\left(\mathbf{X}^{\prime}(\Omega)-j \mathbf{R}^{\prime}\right)}{\operatorname{det}(\mathbf{X}(\Omega)-j \mathbf{R})} .
$$

Analogous expressions, but with different principal submatrices in the numerator, arise for $S_{22}(\Omega)-1$ and $S_{21}(\Omega)$. Specifically, the expression for $S_{22}(\Omega)-1$ involves the upper submatrix of $\mathbf{X}(\Omega)-j \mathbf{R}$, whereas the one for $S_{21}(\Omega)$ uses the upper right submatrix.

It is now evident that the synthesis can be considered in terms of the zeros and poles of expressions related to the scattering parameters and solved via an INEVP involving an alternative set of roots of the characteristic equations for the numerator and denominator. The reference roots can be taken from the formulas defining the scattering parameters in terms of polynomials generated by the filtering function [5]. Alternatively, the reference poles and zeros for the construction of the coupling matrix with AFVC can be determined from the characteristic equations for the numerator and denominator of the filter with frequency-invariant couplings. This links the approach proposed in this paper with synthesis techniques developed in the past for particular configurations, such as the transversal topology that allows for fully-canonical filters [5], resonant-type source-load coupling [14], or frequencydependent $m_{S S}$ and $m_{L L}$ [31]. A filter with a desired characteristic can be synthesised with frequency-invariant couplings and the resulting coupling matrix can be then substituted into (24), providing the reference zeros and poles that are needed in the INEVP_-which is solved for the target coupling topology and frequency-variant inverters.

Finally, it should be remarked upon that, for some cases, the reference eigenvalues may also be obtained directly by computing three sets of eigenvalues of reference coupling matrices with FIIs [32].

\section{Filters With NON-IDEAL ARbitrary Frequency VARIANT INVERTS AND NON-RESONATING NODES}

For the sake of generality, a filter network consisting of $N$ reactances $j X_{i}(\omega)(i=1,2, \ldots, N)$ that are mutually coupled through inverters is considered. The network is connected to the source and load by means of input and output inverters. This gives rise to an $N+2$ network-i.e., $N$ in-series reactances plus the source and load-as shown in Fig. 1.

The series reactances in the filter network, which are frequency-dependent, can be either resonators or nonresonating elements. In particular, the $i$ th reactance can be mathematically expressed as follows:

$$
j X_{i}=j \chi_{i}\left(\omega / \omega_{i 1}-\omega_{i 2} / \omega\right) .
$$

This general formula covers various possibilities for a filter network represented by a coupling diagram with different types of nodes, as follows:

- Resonator-when $\omega_{i 1}=\omega_{i 2}=\omega_{i}$ where $\omega_{i}$ is the resonant frequency and $\chi_{i}$ the reactance slope at $\omega_{i}$.

- Non-resonating reactance-to account for NRNs in the coupling diagram — of different type:

- Inductive NRN with inductance $\chi_{i}$-when $\omega_{i 1}=1$ and $\omega_{i 2}=0$.

- Capacitive NRN with capacitance $\chi_{i}^{-1}$ - when $\omega_{i 1} \rightarrow \infty$ and $\omega_{i 2}=1$.

- Frequency-invariant NRN-when $\omega_{i 1}=\omega$ and $\omega_{i 2}=0$, being zero if $\chi_{i}=0$ is also satisfied.

Note that a combination of a capacitive or inductive and constant values in the NRNs is also admissible.

Non-resonating modes [33] can also be considered by this general synthesis framework, as they can be either treated as resonators with $\omega_{i}$ being away from the pasband or approximated by NRNs. In relation to the formula (13), it should be pointed out that it can be replaced by a more-complex frequency-dependent expression for the node reactance, such as the one associated to a set of resonators-e.g., doublet, triplet, or quadruplet. In this case, the node in the network may be regarded as a higher-level node describing a whole building block that is possibly pre-designed. Note that using building blocks or constituent sections is a popular approach for creating complex filtering networks, and this technique 


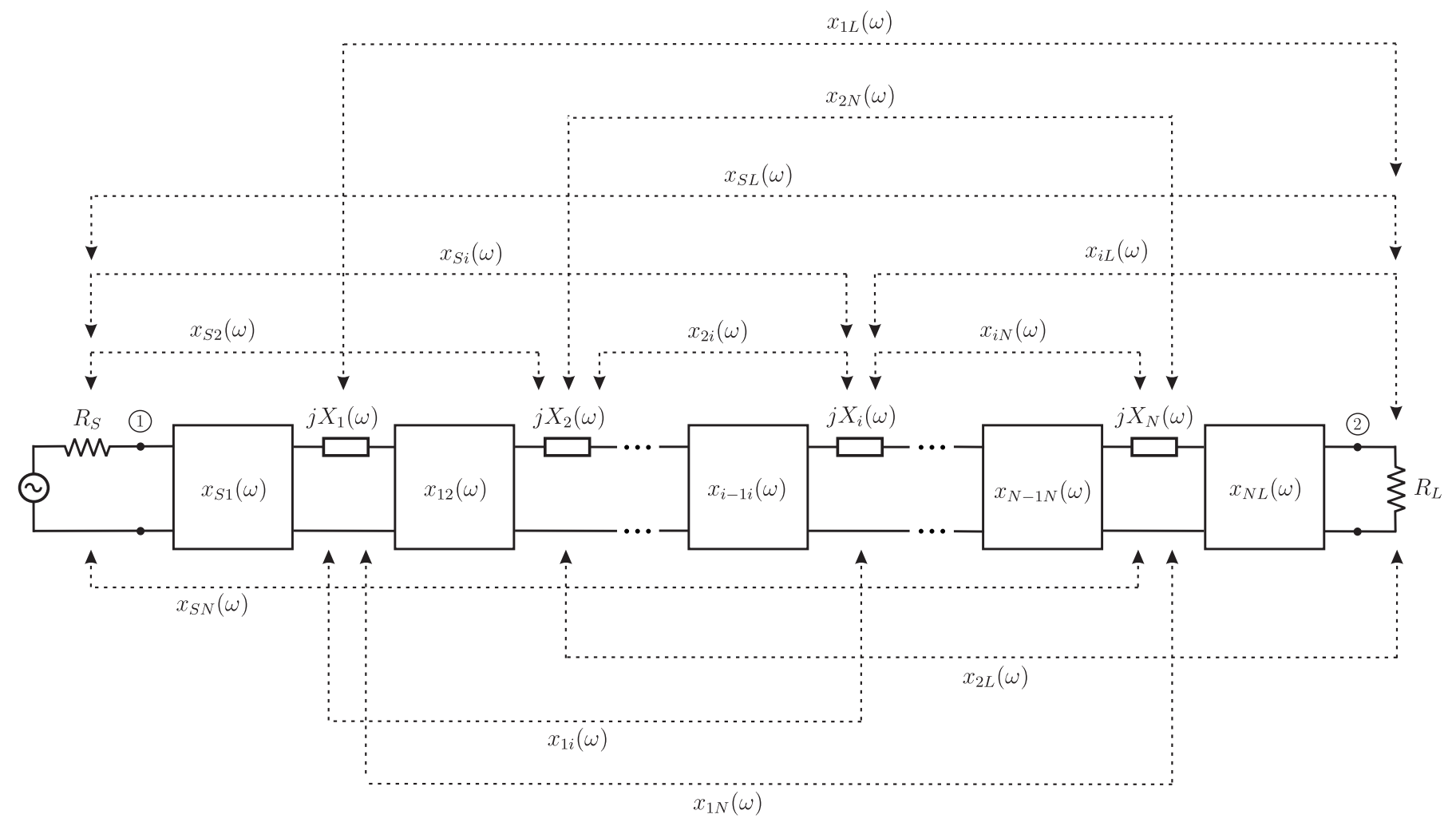

Fig. 1. $N+2$ network with multi-coupled series reactances. All the elements in the network are assumed to be frequency-variant.

can easily be accommodated in the model and the INEVP framework proposed in this paper.

The reactances, as well as the source and load, are all coupled by inverters that can be either constant or frequencyvariant. At this stage, it is assumed that the inverters are ideal in the sense that the diagonal elements of their opencircuit impedance parameters are zero-the modifications to be considered for the frequency-dependent case are discussed later. With regard to the kind of frequency dependence of the off-diagonal elements, three different categories are defined depending on their frequency-derivative behaviour as follows:

1) FII-when the first and higher-order derivatives vanish everywhere.

2) LFVI-when the first derivative is nonzero, and the second and higher-order derivatives vanish everywhere.

3) AFVI - when the second derivative or any higher-order derivative is nonzero.

To denote the different categories of nodes and inverters to be used in a coupling-routing diagram, the representation convention of symbols depicted in Fig. 2 is adopted here.

The reactance matrix $\mathbf{X}(\omega)$ for the network shown in Fig. 1 has two extra rows and columns-so its dimension is $(N+$ 2) $\times(N+2)$-as follows:

$$
\mathbf{X}(\omega)=\left[\begin{array}{cccccc}
x_{S S} & x_{S 1} & x_{S 2} & \cdots & x_{S N} & x_{S L} \\
x_{S 1} & x_{11} & x_{12} & \cdots & x_{1 N} & x_{1 L} \\
\vdots & \vdots & \vdots & \ddots & \vdots & \vdots \\
x_{S N} & x_{1 N} & x_{2 N} & \cdots & x_{N N} & x_{N L} \\
x_{S L} & x_{1 L} & x_{2 L} & \cdots & x_{N L} & x_{L L}
\end{array}\right] .
$$

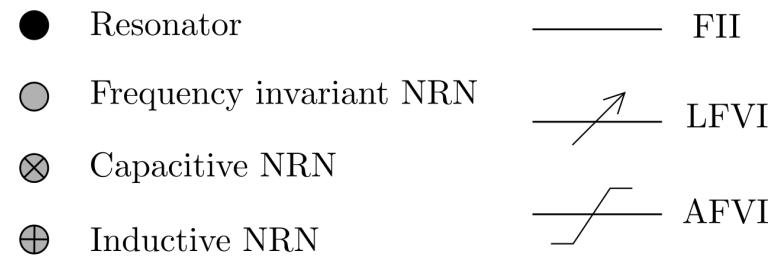

Fig. 2. Convention of symbols of nodes and inverters used in coupling-routing diagrams.

The diagonal entries in this matrix are given by $j x_{i i}=j X_{i}$. For practical reasons, it is convenient to normalize the coupling matrix as it is done in the conventional synthesis of the frequency-invariant coupling matrix in the prototype domain. To normalize rows and columns, the source $R_{S}$ and load $R_{L}$ resistances, the fractional bandwidth $\Delta$, and the parameter $\chi$ (reactance slope for resonators and inductance or inverse of capacitance for inductive or capacitive NRNs, respectively) are used. The normalization process is carried out by left and right multiplying the coupling matrix by diagonal matrices $\mathbf{Q}$, where the diagonal entries of the $\mathbf{Q}$ matrix are given by

$$
\begin{aligned}
q_{S S} & =R_{S}^{-1 / 2}, \quad q_{L L}=R_{L}^{-1 / 2} \\
q_{i i} & =\left\{\begin{array}{cc}
\chi_{i}^{-1 / 2} \Delta^{-1 / 2} & \text { when } \chi_{i} \neq 0 \\
1 & \text { otherwise }
\end{array} .\right.
\end{aligned}
$$

After introducing this normalization, the following expression is obtained for the extended coupling matrix:

$$
\mathbf{M}=\mathbf{Q X}(\omega) \mathbf{Q} .
$$


Besides, for the termination impedance matrix $\mathbf{R}$ in (12), it is also normalized. Observe that when all the inverters are frequency-invariant, all the coupled reactances are considered to be identical-i.e., synchronously-tuned resonators with $\omega_{0 i}=\omega_{0}$ - and no NRNs are present in the filter network, the coupling matrix adopts the well-known form that is commonly used in the traditional synthesis in the lowpassprototype domain. In addition, it is noteworthy to notice that the normalization process does not affect the localization of the roots of the characterization equation, so that the diagonal elements of matrix $\mathbf{Q}$ can be chosen differently. For instance, the normalization for NRNs can be performed as in [34].

Note that the model introduced here is much more general than the one proposed in [6] — which only addressed couplings that are either constant or inversely proportional to frequency - or the one in [7]—where a combination of frequency-dependent couplings proportional or inversely proportional to frequency (either capacitive or inductive) was considered. Such constraints on the type of frequency dependence for couplings are overcome by the proposed synthesis method.

\section{A. Loading Effect Due to Non-Ideal Inverters}

The matrix derived above can be used in the inverse eigenvalue problem to find the parameters that are required to fulfill the filter specification. It is, however, convenient to take into account the effect of the loading reactances on the main diagonal when the inverters are non-ideal. By a nonideal inverter, it is understood a two-port coupling network characterized by its open-circuit impedance parameters; nevertheless, unlike for ideal inverters, these coupling networks have nonzero diagonal entries in their impedance-parameter description. For example, if the coupling between reactances $i$ and $k$ is considered, the coupling network associated to it can be described by the following open-circuit impedance matrix:

$$
\mathbf{Z}_{i k}=\left[\begin{array}{cc}
z_{i k}^{11} & z_{i k}^{12} \\
z_{i k}^{12} & z_{i k}^{22}
\end{array}\right]
$$

The diagonal elements will load both reactances, and this can be taken into account in the coupling-matrix definition. Note that each reactance can be coupled to other reactances, so that all the non-ideal inverters that load each reactance considered. Like for ideal inverters, frequency-invariant (i.e., constant) couplings (FICs) and the two categories of dispersive couplings-LFVCs and AFVCs_-are distinguished. As noted above, in order to compensate for the loading effect due to any of these couplings, the reactances will have to be modified. It is useful to reflect this effect in the coupling matrix, so that the loading effect is expressed in an explicit way. To this end, the coupling matrix is decomposed into three parts, as follows:

$$
j \mathbf{X}(\omega)=j \mathbf{X}^{x}+j \mathbf{X}^{l}+j \mathbf{X}^{I}
$$

where $\mathbf{X}^{x}$ is the diagonal matrix of reactances, $\mathbf{X}^{l}$ is the diagonal matrix that accounts for the cumulative loading effect due to all the non-ideal inverters, and $\mathbf{X}^{I}$ is a matrix with zeros on the diagonal that describes the coupling provided by ideal inverters, in general with arbitrary frequency variation:

$$
\mathbf{X}^{x}=\operatorname{diag}\left[X_{S}, X_{1}, X_{2}, \cdots X_{N}, X_{L}\right]
$$

$$
\mathbf{X}^{l}=\operatorname{diag}\left[x_{S S}^{l}, x_{11}^{l}, \cdots x_{N N}^{l}, x_{L L}^{l}\right]
$$

with

$$
j x_{k k}^{l}=\sum_{l=k+1}^{N+2} z_{k l}^{11}+\sum_{l=0}^{k-1} z_{k l}^{22}
$$

and

$$
j x_{i k}^{I}=-z_{i k}^{12}, \quad i \neq k \quad j x_{k k}^{I}=0 .
$$

Note that in the above equations $i, k=0,1, \ldots N+1$ and, for the sake of compactness in (22) and (23), $i, k$ or $l=0$ is assigned to the source and $i, k$ or $l=N+1$ refers to the load.

The above expression defines the component of the nonnormalized coupling matrix. Practical computation involves normalized-component matrices and the entire normalized coupling matrix is

$$
\mathbf{M}=\mathbf{Q X}(\omega) \mathbf{Q}=\mathbf{M}^{x}+\mathbf{M}^{l}+\mathbf{M}^{I} .
$$

As a final remark, note that the elements of the matrix $\mathbf{M}^{l}$ are not optimizable when the IEVP is solved. This is because their values are a function of variables related to the offdiagonal elements of the open-circuit impedance parameters of the inverters contained in the matrix $\mathbf{X}^{I}$. Consequently, entries in $\mathbf{M}^{l}$ change implicitly as a result of variations in $\mathbf{M}^{I}$.

\section{B. Coupled-Resonator Filter Synthesis Step by Step}

Given the filter specifications (bandwidth, center frequency, return-loss level, location of TZs, filter order $n$, number of nodes $N$ and network topology, formulas for reactances and characteristics of the AFVC elements-non-ideal AFVI-, and a chosen reference model as discussed in the previous section-e.g., short-circuit admittance parameters, scattering parameters, or eigenvalues of the reference frequency-invariant network), the following steps have to be executed assuming that the reference model is associated with the poles and zeros of $y_{11}$ and $y_{22}$ or $S_{11}-1$ and $S_{22}-1$ :

Step 1. Based on the desired transmission characteristics and the filter order, synthesise three polynomials for the numerators and denominator of the reference filternetwork model.

Step 2. Define three sets of reference roots as follows: $\boldsymbol{\omega}_{p}$ with the reference poles associated with eigenvalues of the entire target coupling matrix, $\boldsymbol{\omega}_{u}$ with the reference zeros associated to the eigenvalues of the upper principal submatrix of the target filter model, and $\boldsymbol{\omega}_{l}$ with the reference zeros associated to the eigenvalues of the lower principal submatrix.

Step 3. Evaluate the normalization coefficients for the rows and columns of the coupling matrix.

Step 4. Solve the INEVP by optimization. The goal function for the optimization is given by

$$
C=\left\|\boldsymbol{\lambda}_{u}-\boldsymbol{\omega}_{u}\right\|+\left\|\boldsymbol{\lambda}_{l}-\boldsymbol{\omega}_{l}\right\|+\left\|\boldsymbol{\lambda}-\boldsymbol{\omega}_{p}\right\|
$$

where $\boldsymbol{\lambda}, \boldsymbol{\lambda}_{u}$, and $\boldsymbol{\lambda}_{l}$ are vectors of eigenvalues of the coupling matrix and its two principal submatrices.

Step 5. Denormalize the elements of the coupling matrix to get the values of the reactance part of the network as

$$
\mathbf{X}=\mathbf{Q}^{-1} \mathbf{M}(\omega) \mathbf{Q}^{-1}
$$


Some additional remarks to be considered are given below.

Remark 7. The choice of the reference sets is somehow arbitrary. They can be selected according to the needs, and they can also be the roots associated with bottom left or upper right principal submatrices (e.g., for $S_{21}$ ) or even as central principal submatrix (poles of $y_{11}, y_{21}$, and $y_{22}$ in the extended coupling matrix). Note also that for lossy networks-which are not discussed in this work-, a total of four sets is needed.

Remark 8. Due to the presence of AFVCs or NRNs, the size of the coupling matrix to be synthesized that is $(N+2) \times(N+2)$ is not equal to $(n+2) \times(n+2)$, where $n$ is the degree of the network. In fact, as it will be seen in the synthesis examples provided in Section $\mathrm{V}$, both $n>N$ and $n<N$, as well as $n=N$, are possible.

Remark 9. The INEVP is solved in the passband domain, but the synthesis of the polynomials can be carried out in the lowpass filter domain and, then, the reference sets can be transformed to the passband domain. This is recommended as the polynomial synthesis in the lowpass domain is more stable numerically than in the passband domain.

Remark 10. The reference polynomials have to be known; for instance, these polynomials can be synthesised analytically or by optimization. Examples include polynomials for filtering functions involving arbitrary locations of TZs [6], [7] or even functions with $n+1$ TZs that can be achieved by adding a resonant-type source-load coupling [14].

Remark 11. During the optimization process needed to obtain the INEVP solution, splitting (24) may be used so that each design parameter can be altered independently. The optimizable parameters can be related to reactances at the filter nodes (e.g., resonant frequency $\omega_{0 i}$ ) and to the coupling networks (parameters controlling the off-diagonal elements of the frequency-variant couplings $\mathbf{M}^{I}$ ). As noted before, the diagonal elements of the couplings, which are collected in the matrix $\mathbf{M}^{l}$, are in general not optimizable, and they change whenever the elements of $\mathbf{M}^{I}$ are altered. It is also possible to assign the parameter $\chi_{i}$ in some or all nodes as an optimization variable. In this case, the element of the normalization matrix $\mathrm{Q}$ in (16) corresponding to this node needs to be replaced by an arbitrary scaling constant $\sqrt{\chi_{i}^{0}}$.

Remark 12. The synthesis procedure will not converge in the following situations:

1) The desired specification cannot be achieved with the assumed coupling scheme and type of frequency-variation profile for the open-circuit impedance parameters of the coupling networks.

2) The number of eigenvalues of the coupling matrix or its principal submatrices is different from the number of reference zeros and poles.

\section{ARbitraRy FrequenCy-VARIANT COUPLINGS}

As previously mentioned, the proposed synthesis framework is fully general for the design of microwave filters with coupling networks exhibiting arbitrary frequency-variant profiles. Nevertheless, for illustration purposes of the engineered filter synthesis methodology, three particular cases of frequencyvariant coupling topologies are considered here.

The circuit configurations of the three selected examples of AFVC networks are depicted in Fig. 3. As can be seen, they consist of reciprocal two-port circuits-which are also symmetrical for the cases provided in Fig. 3(a) and (b)-, whose electrical behavior can be represented by means of their impedance-parameter matrix as follows:

- Type I: shunt impedance $Z_{a}=Z_{a}(\omega)$-i.e., Fig. 3(a):

$$
Z_{11}(\omega)=Z_{22}(\omega)=Z_{21}(\omega)=Z_{a} .
$$

- Type II: two shunt impedances $Z_{a}=Z_{a}(\omega)$ and $Z_{b}=$ $Z_{b}(\omega)$ that are connected in parallel-i.e., Fig. 3(b):

$$
Z_{11}(\omega)=Z_{22}(\omega)=Z_{21}(\omega)=\frac{Z_{a} Z_{b}}{Z_{a}+Z_{b}} .
$$

- Type III: two shunt impedances $Z_{a}(\omega)$ and $Z_{b}(\omega)$ that are separated by a transmission-line segment with characteristic impedance $Z_{C}=1 / Y_{C}$ and frequencydependant electrical length $\theta_{C}=\beta(\omega) l$ (where $\beta(\omega)$ and $l$ are the phase constant and the physical length of the transmission-line segment, respectively)—i.e., Fig. 3(c):

$$
\begin{aligned}
Z_{11}(\omega) & =\frac{Z_{a} Z_{b}+j Z_{a} Z_{C} \tan \theta_{C}}{\left(Z_{a}+Z_{b}\right)+j\left(Z_{C}+Y_{C} Z_{a} Z_{b}\right) \tan \theta_{C}} \\
Z_{22}(\omega) & =\frac{Z_{a} Z_{b}+j Z_{b} Z_{C} \tan \theta_{C}}{\left(Z_{a}+Z_{b}\right)+j\left(Z_{C}+Y_{C} Z_{a} Z_{b}\right) \tan \theta_{C}} \\
Z_{21}(\omega) & =\frac{Z_{a} Z_{b} / \cos \theta_{C}}{\left(Z_{a}+Z_{b}\right)+j\left(Z_{C}+Y_{C} Z_{a} Z_{b}\right) \tan \theta_{C}} .
\end{aligned}
$$

Note that if $\theta_{C}=0$, then the Type-III coupling network becomes the Type-II one.

It should be remarked upon that these three types of frequency-variant coupling networks can exhibit different behavior in terms of transmission-zero and pole generation. For example, if the impedances $Z_{a}$ and $Z_{b}$ are realized by means of open-ended transmission-line segments or stubs so that

$$
\begin{aligned}
& Z_{a}(\omega)=-j Z_{A} / \tan \left(\omega \theta_{A 0} / \omega_{0}\right) \\
& Z_{b}(\omega)=-j Z_{B} / \tan \left(\omega \theta_{B 0} / \omega_{0}\right)
\end{aligned}
$$

where $Z_{A}$ and $Z_{B}$ are the characteristic impedances and $\theta_{A 0}$ and $\theta_{B 0}$ are the electrical lengths at $\omega_{0}$ of the transmissionline segments, then the following features are obtained for the coupling networks in Fig. 3 that can be adjusted independently:

- Type I: one TZ at $\omega_{z}=\omega_{0} \pi /\left(2 \theta_{A 0}\right)$.

- Type II: two TZs at $\omega_{z 1}=\omega_{0} \pi /\left(2 \theta_{A 0}\right)$ and $\omega_{z 2}=$ $\omega_{0} \pi /\left(2 \theta_{B 0}\right)$ with one pole between them at $\omega_{p}$ so that $Z_{a}\left(\omega_{p}\right)=-Z_{b}\left(\omega_{p}\right)$.

- Type III: two TZs at $\omega_{z 1}=\omega_{0} \pi /\left(2 \theta_{A 0}\right)$ and $\omega_{z 2}=$ $\omega_{0} \pi /\left(2 \theta_{B 0}\right)$, being both below or above one pole at $\omega_{p}$ or even each of them at one different side with regard to the pole location as for the case $\theta_{C}=0$.

For illustration purposes, Fig. 4 shows examples of the transimpedance parameter $Z_{21}$ (imaginary part) for the three types of frequency-variant coupling networks in Fig. 3, where the aforementioned properties in terms $\mathrm{TZ}$ and pole generation 


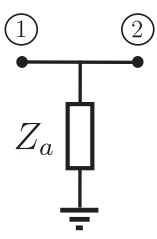

(a)

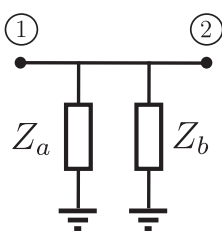

(b)

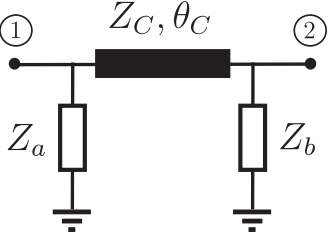

(c)
Fig. 3. Examples of two-port circuits corresponding to AFVC networks (the impedances $Z_{a}$ and $Z_{b}$ and the electrical length $\theta_{C}$ of the transmissionline segment are assumed to be frequency-dependent). (a) Type I: shunt impedance. (b) Type II: two in-parallel shunt impedances. (c) Type III: two shunt impedances separated by a transmission-line segment.

are verified. Note also that, due to the inherent frequencyperiodic behavior of the transmission-line segments building these coupling networks, additional TZs and poles are created at other frequency ranges, despite their spectral positions can not be controlled independently.

\section{SYNTHESIS EXAMPLES}

This section presents the synthesis results of seven different bandpass filter architectures using a variety of frequencydependent couplings or NRNs. The provided examples include six inline topologies and one quadruplet. In all these examples, the reference poles and zeros were determined from the targeted filtering functions, which were constructed using the analytical procedure described in [5]. The coupling matrix was split as indicated in (24). In all those examples that involve dispersive couplings (either LFVC or AFVC of Type-I, TypeII, and Type-III), nonzero diagonal elements in their opencircuit impedance-parameter matrices were introduced as previously discussed. Consequently, when solving the associated INEVP, the resonant frequencies $\omega_{i}$ in (13) in the matrix $\mathbf{M}^{x}$ were exploited as optimizable parameters to compensate for the loading effect of the imperfect inverters (matrix $\mathbf{M}^{l}$ ). The elements of the matrix $\mathbf{M}^{I}$ were also considered optimizable variables, while the entries of the matrix $\mathbf{M}^{l}$ changed only implicitly as a result of modifications in $\mathbf{M}^{I}$.

\section{A. Third-Order Filters With Two Transmission Zeros}

To illustrate the capabilities of the proposed synthesis method based on INEVP for various types of dispersive couplings and topologies involving NRNs, five distinct examples of synthesized third-order bandpass filters with two TZs are provided here. In each case, it is assumed that the center frequency and bandwidth is $2.4 \mathrm{GHz}$ and $0.15 \mathrm{GHz}$ (i.e., $6.25 \%$ in relative terms), respectively, while the minimum inband return-loss level is $20 \mathrm{~dB}$. For the first-to-four filters, the TZs are placed at $2.26 \mathrm{GHz}$ and $2.62 \mathrm{GHz}$ (i.e., at both sides of the passband). Specifically, the four configurations considered for these four bandpass filter examples are as follows:

- Three resonators with two LFVCs: Fig. 5(a).

- Three resonators with two Type-I AFVCs: Fig. 5(b).

- Four resonators with one NRN: Fig. 5(c).

- Two resonators with one Type-II AFVC: Fig. 5(d).

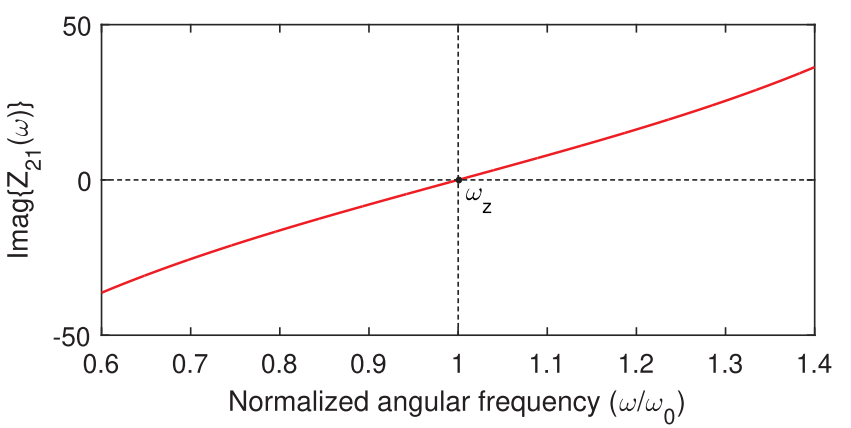

(a)

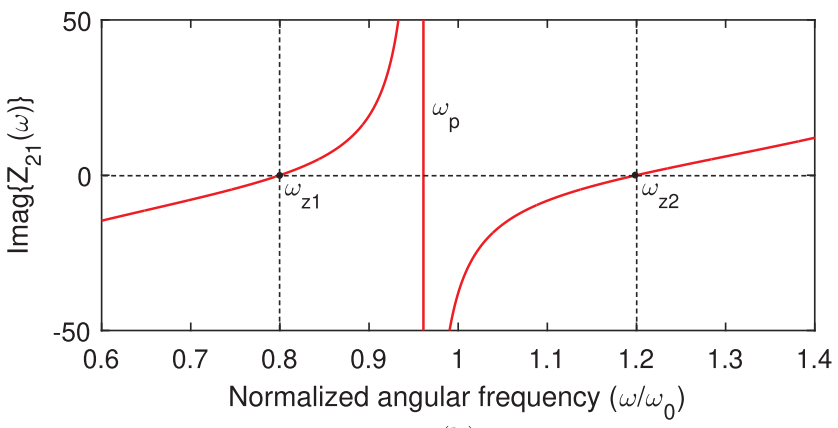

(b)

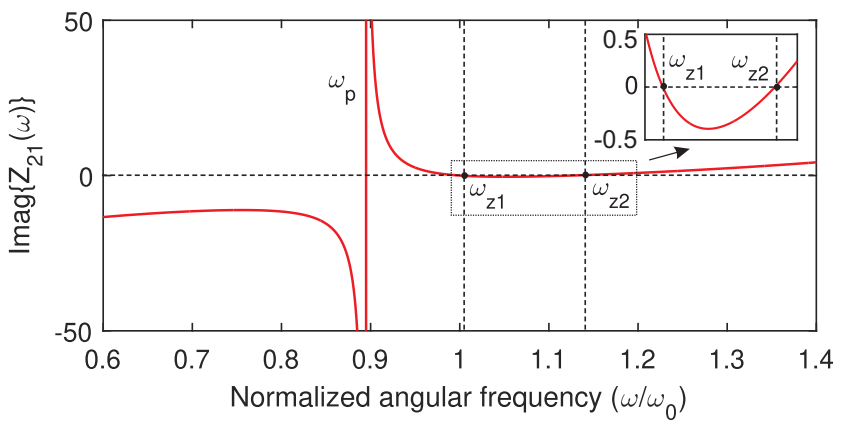

(c)

Fig. 4. Examples of $Z_{21}$ parameter-imaginary part-for the frequencyvariant coupling networks in Fig. $3\left(Z_{0}=50 \Omega\right)$. (a) Type-I coupling network in Fig. 3(a) $\left(Z_{A}=Z_{0}\right.$ and $\left.\theta_{0 A}=\pi / 2\right)$. (b) Type-II coupling network in Fig. 3(b) $\left(Z_{A}=Z_{0}, \theta_{0 A}=0.625 \pi, Z_{B}=Z_{0}\right.$, and $\left.\theta_{0 B}=0.4167 \pi\right)$. (c) TypeIII coupling network in Fig. 3(c) - both transmission zeros are produced above the pole: $\left(Z_{A}=0.78 Z_{0}, \theta_{0 A}=\pi / 2, Z_{B}=0.4 Z_{0}, \theta_{0 B}=0.4444 \pi\right.$, $\left.Z_{C}=1.3 Z_{0}, \theta_{0 C}=0.0722 \pi\right)$.

In the fifth bandpass filter example, the two $\mathrm{TZ}$ are positioned above the passband at $2.62 \mathrm{GHz}$ and $2.82 \mathrm{GHz}$, for which two resonators that produce two poles and one Type-III AFVC that generates the two TZs and one pole are employed. The coupling-routing topology for this filter is shown in Fig. 5(d). Hence, all three types of coupling networks depicted in Fig. 3 are considered in these filter examples.

As can be inferred from the description above, even though the specification for the bandpass filter example with two TZs at both sides of the passband is the same, the way in which the TZs are obtained in each design is different. In particular, the dispersive couplings exhibit different frequency-variation pattern. For reference, the first filter example is synthesised in the lowpass-prototype domain by using the assumption of linear frequency dependence for the inverters, and the procedure described in [13] and [15] is used. It involves the 
TABLE I

Resonant Frequencies for Nodes $f_{i}=\frac{\omega_{i}}{2 \pi}$ (In GHz), Stub Impedances (IN $\Omega$ ) And Their Electrical Lengths at $f_{0}$ (In DegRees) (THIRD-ORDER BANDPASS FILTERS WITH TWO TZs)

\begin{tabular}{|l||c|c|c|c|c|c|c|c|c|c|c|}
\hline Topology in Fig. 5 & $f_{1}$ & $f_{2}$ & $f_{3}$ & $f_{4}$ & $f_{5}$ & $Z_{s 1}$ & $Z_{s 2}$ & $\theta_{1}$ & $\theta_{2}$ & $Z_{C}$ & $\theta_{C}$ \\
\hline (a) LFVC & 2.446 & 2.395 & 2.328 & - & - & 153.096 & 92.454 & 95.575 & 82.442 & - & - \\
\hline (b) Type-I AFVC & 2.446 & 2.395 & 2.329 & - & - & 158.716 & 89.394 & 95.602 & 82.552 & - & - \\
\hline (c) NRN & 2.387 & - & 2.620 & 2.260 & 2.387 & - & - & - & - & - & - \\
\hline (d) Type-II AFVC & 2.387 & 2.387 & - & - & - & 80.330 & 48.337 & 95.600 & 82.540 & - & - \\
\hline (d) Type-III AFVC & 2.372 & 2.400 & - & - & - & 27.569 & 13.894 & 82.446 & 76.596 & 83.705 & 4.012 \\
\hline
\end{tabular}

TABLE II

VAlues of Frequency-InVARIANt COUPlings (Third-Order BandPass Filters With Two TZs)

\begin{tabular}{|l|l|l|l|l|l|l|}
\hline Topology in Fig. 5 & $m_{S 1}$ & $m_{N L}$ & $m_{12}$ & $m_{23}$ & $m_{24}$ & $m_{25}$ \\
\hline (a) LFVC & 1.415 & 1.327 & - & - & - & - \\
\hline (b) Type-I AFVC & 1.416 & 1.327 & - & - & - & - \\
\hline (c) NRN & 1.178 & 1.178 & 0.809 & 1.197 & 0.891 & 0.809 \\
\hline (d) Type-II AFVC & 1.178 & 1.178 & - & - & - & - \\
\hline (d) Type-III AFVC & 1.147 & 1.142 & - & - & - & - \\
\hline
\end{tabular}

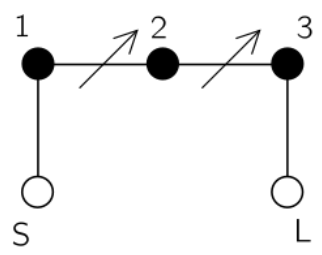

(a)

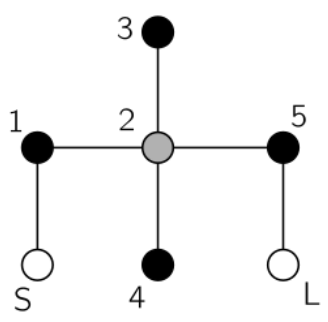

(c)

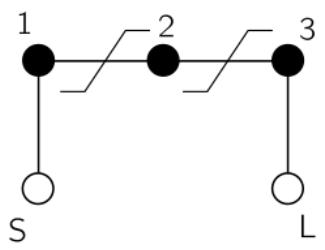

(b)

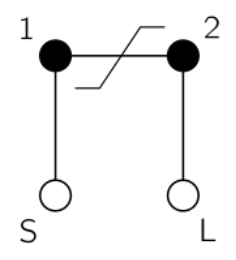

(d)
Fig. 5. Coupling-routing diagrams for the third-order bandpass filter examples. (a) Three resonators with two LFVCs. (b) Three resonators with two AFVCs. (c) Four resonators with one NRN. (d) Two resonators with one AFVC.

solution of the generalized IEVP in (2). In all other cases, the synthesis is performed in the bandpass domain and the new INEVP framework outlined above is employed. The final design parameter values resulting from the synthesis for all these third-order bandpass filter examples are given in Tables I and II, while their power transmission and reflection responses derived from the synthesis process are compared in Fig. 6. In this figure, the representation of these responses in a broad frequency interval is given, whereas the inset shows their detail in the vicinity of the passband. As demonstrated, between $2 \mathrm{GHz}$ and $2.8 \mathrm{GHz}$, the coupling-matrix responses of the four examples exactly match the filter specifications (characterization obtained directly from the rational representation of the prototype response). The differences among them are clearly visible above $3 \mathrm{GHz}$, where the characteristics for the designs involving AFVCs deviate from the desired one.

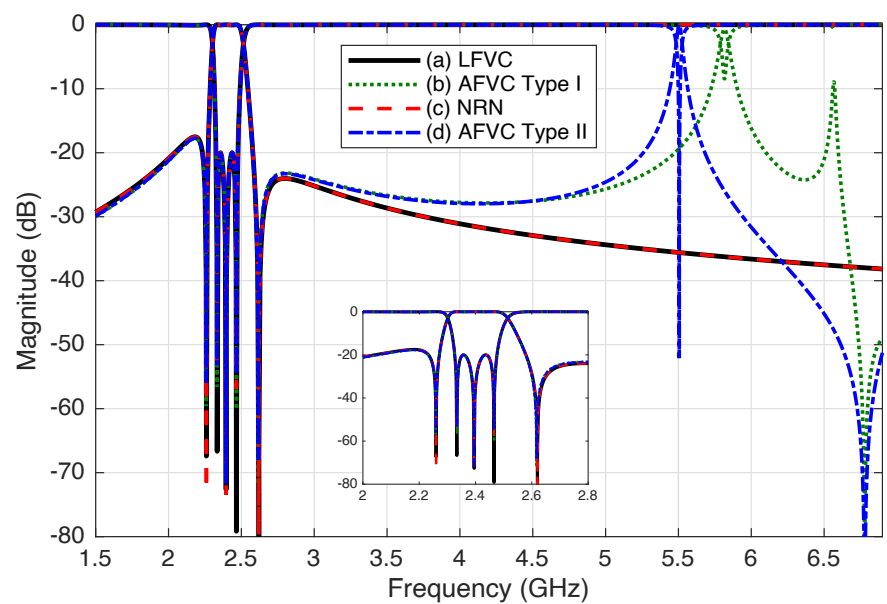

Fig. 6. Power transmission $\left(\left|S_{21}\right|\right)$ and reflection $\left(\left|S_{11}\right|\right)$ responses of the third-order bandpass filter examples with two TZs (one TZ at each passband side) computed from the synthesized coupling matrix for four different implementations involving FVCs or NRNs: Fig. 5(a)-(d) - the inset shows the responses near the passband range.

This is a natural consequence of the nonlinear and periodic frequency-variation patterns of the inverter parameters. It has to be pointed out that the results given in Table I clearly show that the resontors are detuned, as a result of the loading effect due to the non-ideal inverters.

Note that the results given in Tables I and II show that the designs involving three resonators and two FVCs (i.e., Fig. 5(a) and (b)) differ, even though the dispersive couplings are implemented as shunt open-ended stubs in both exampleseach stub is quarter-wavelength long at the TZ frequency generated by it, and shows a non-linear frequency-variation profile in its equivalent impedance. For the sake of illustration, in the coupling scheme in Fig. 5(a), such impedance was approximated by a linear function of frequency-which is a relatively accurate approximation for this type of stub-, where its slope is determined by the characteristic impedance. In the second design (i.e., Fig. 5(b)), the stub impedance was not approximated by a linear function of frequency. Thus, the stubs were modeled as AFVCs with the Type-I coupling 


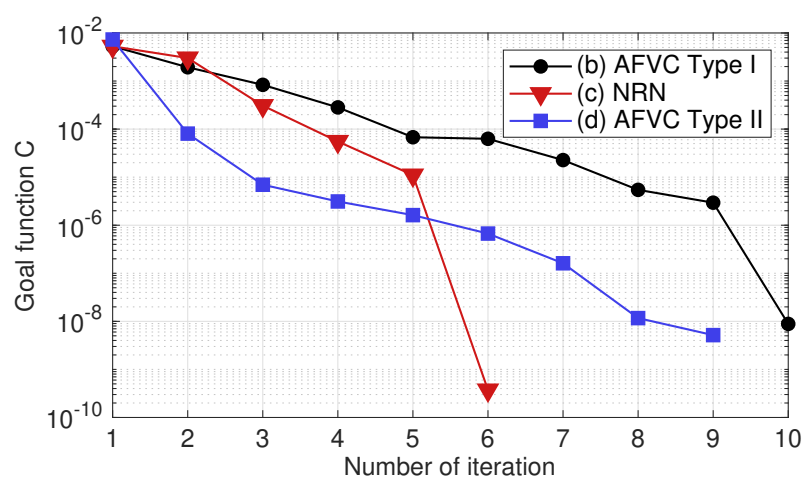

Fig. 7. Convergence of the optimization procedure for solving the INEVPthird-order bandpass filter examples with two TZs at both sides of the passband.

network. Accordingly, the synthesis was carried out in this case by considering an INEVP. After reaching convergence, the values for the stub impedances were found to be $158.72 \Omega$ and $89.40 \Omega$. This result is slightly different from what was obtained by linearization ( $153.10 \Omega$ and $92.45 \Omega$ ), and this is due to the different models of the frequency-variant inverters.

The implementations involving four resonators and one NRN (i.e., Fig. 5(c)) and two resonators with one Type-II AFVC (i.e., Fig. 5(d)) lead to designs with identical $m_{S 1}$ and $m_{N L}$. In fact, the middle section in the design with one NRN produces one pole and two TZs [35]. They are created at exactly at the same locations as for the Type-II AFVC. However, whereas the locations of the TZs in the NRN section are the same as the resonant frequencies of resonators 3 and 4 , they are determined by the lengths of the stubs in the AFVC.

Concluding the discussion related to the synthesis of the bandpass filter examples with two TZs at both sides of the passband, two major points must be highlighted. First, the convergence of INEVP process solution in all these cases was found to be very quick. This is demonstrated in Fig. 7. It has to be noted, however, that the number of iterations depends on the starting point. For the results presented in Fig. 7, the initial values were quite far from the final ones (which is evident from the high value of the goal function at the first iteration, as shown in the plot). The second aspect is the number of eigenvalues $(M)$ versus the number of nodes $(N)$ in the network and the filter order $n(n=3$ in all the examples considered above). For the first structure with two LFVCs, the number of finite eigenvalues of the coupling matrix matches the number of nodes (three in this case) so that $M=N=n$. In the implementation involving Type-I AFVCs, $M=N=n$ is also satisfied; however, the number of eigenvalues of the INEVP depends on the frequency range-it is three when the interval is not too wide (e.g., $1.5-3 \mathrm{GHz}$ ) and increases to five when the frequency range is extended up to $7 \mathrm{GHz}$. For the structure with the NRN, $N=5$ and $M=5$, while $n=3$ (i.e., $M=N>n$ ); here, the two extra eigenvalues are at infinity and the number of eigenvalues is the same regardless of the considered frequency range. In the implementation involving two resonators and one Type-II AFVC, there are two nodes in the network while the filter

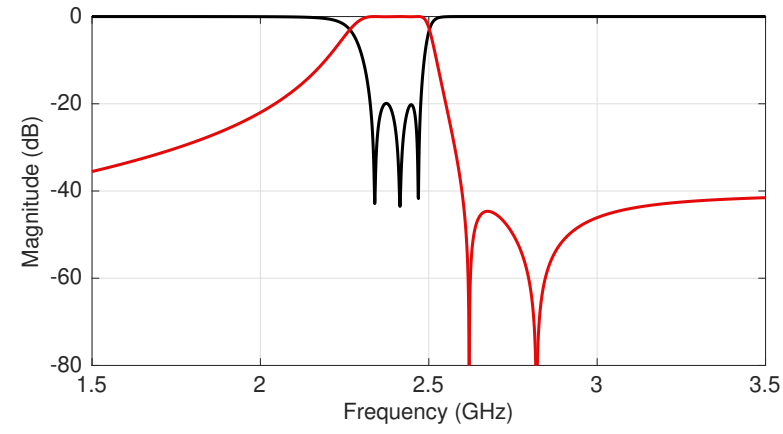

Fig. 8. Power transmission $\left(\left|S_{21}\right|\right)$ and reflection $\left(\left|S_{11}\right|\right)$ responses of the third-order bandpass filter example with two TZs above the passband using one Type-III AFVC: Fig. 5(d).

order is three, so that $n>N$. In such case, the INEVP gives three eigenvalues when considering the frequency range 1.5$3 \mathrm{GHz}(M=n>N)$ and four when the upper limit is set to $7 \mathrm{GHz}$, thus resulting in $M>n>N$.

The last example of third-order bandpass filter is an inline filter with one Type-III AFVC (i.e., Fig. 5(d)). In this case, both TZs were imposed to be placed above the passband, as it can be seen in the filter transfer function drawn in Fig. 8. The optimization procedure converged in 17 steps for this example. The final result of the optimization is given in Tables I and II.

\section{B. Higher-Order Filters With AFVCs and NRNS}

As more-challeging examples, the synthesis of higher-order bandpass filters with multiple TZs is subsequently addressed.

The first example consists of a fifth-order bandpass filter with four resonators and one Type-II AFVC. Similarly to the previous example of the third-order bandpass filter with one Type-II AFVC, the number of nodes is smaller that the order of the filter (i.e., $N<n$ ). The center frequency is $3 \mathrm{GHz}$, the absolute bandwidth is $120 \mathrm{MHz}$ (i.e., fractional bandwidth equal to $4 \%$ ), the minimum in-band return-loss level is 20 $\mathrm{dB}$, and its three TZs are imposed to be positioned at 2.85 $\mathrm{GHz}, 3.12 \mathrm{GHz}$, and $3.25 \mathrm{GHz}$. Its coupling-routing diagram is shown in Fig. 9(a), which corresponds to a quadruplet with one cross-coupling between resonators 1 and 4. In this network, two symmetrically-located TZs are inherently produced by the quadruplet with FIIs. In addition, by replacing the coupling between resonators 2 and 3 with the Type-II AFVC, the filter order is increased by one and one additional TZ is created above the passband. Note also that there is no symmetry in the location of TZs. In this case, the solution of the INEVP was obtained after 21 iterations, leading to the following final solution: $f_{1}=f_{4}=2.999 \mathrm{GHz}, f_{2}=f_{3}=3.008 \mathrm{GHz}$, $Z_{s 1}=10.861 \Omega, Z_{s 2}=31.631 \Omega, \theta_{1}=96.819^{\circ}, \theta_{2}=$ $87.013^{\circ}, m_{S 1}=m_{4 L}=1.001, m_{12}=m_{34}=0.889$, and $m_{14}=0.018$. The response of the filter is shown in Fig. 10(a).

The second example is another fifth-order bandpass filter, but in this case its associated coupling network comprises a total of seven resonators and two NRNs. The coupling-routing diagram of the filter is shown in Fig. 9(b). Each set shaped by a NRN and its two adjoining resonators produces one pole and two TZs. The filter 


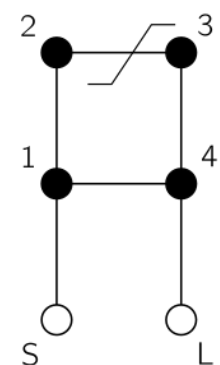

(a)

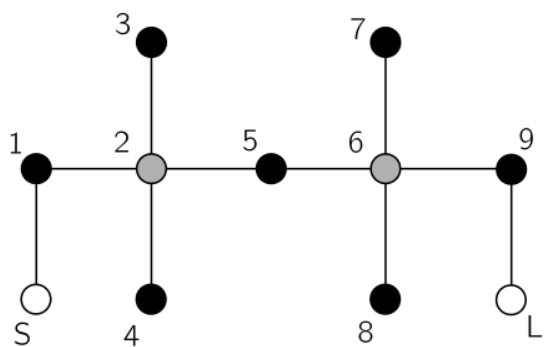

(b)
Fig. 9. Coupling-routing diagrams for the fifth-order bandpass filter examples (a) Four resonators with one AFVC. (b) Seven resonators with two NRNs.

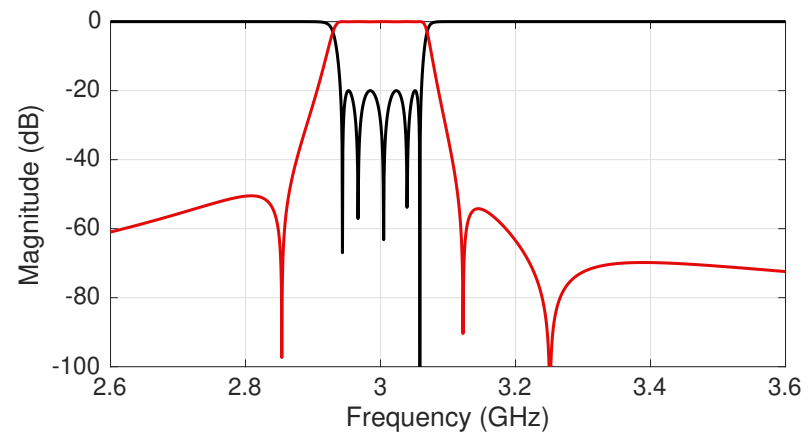

(a)

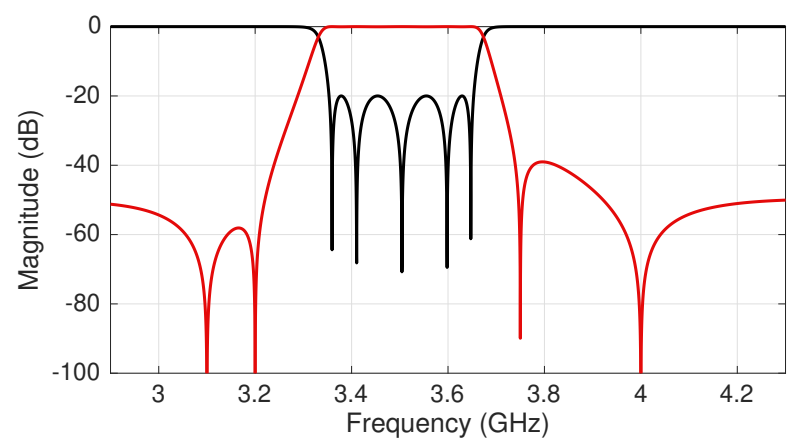

(b)

Fig. 10. Power transmission $\left(\left|S_{21}\right|\right)$ and reflection $\left(\left|S_{11}\right|\right)$ responses of the fifth-order bandpass filter examples. (a) Quadruplet with one Type-II AFVC. (b) Seven resonators with two NRNs.

is centered at $3.5 \mathrm{GHz}$, its bandwidth is equal to 300 $\mathrm{MHz}$ (i.e., $8.57 \%$ in relative terms), its minimum in-band return-loss level is equal to $20 \mathrm{~dB}$, and the positions of its four TZs are set at $3.1 \mathrm{GHz}, 3.2 \mathrm{GHz}, 3.75 \mathrm{GHz}$, and $4 \mathrm{GHz}$. The final result of the optimization is as follows: $f_{1}=3.495 \mathrm{GHz}, f_{3}=101 \mathrm{GHz}, f_{4}=4.000 \mathrm{GHz}$, $f_{5}=3.506 \mathrm{GHz}, f_{7}=3.200 \mathrm{GHz}, f_{8}=3.750 \mathrm{GHz}$, $f_{9}=3.526 \mathrm{GHz}, m_{S 1}=1.051, m_{12}=0.470, m_{23}=1.024$, $m_{24}=1.088, m_{25}=0.355, m_{56}=0.533, m_{67}=0.984$, $m_{68}=0.783, m_{69}=0.781$, and $m_{9 L}=1.159$. Fig. 10(b) shows the response of the filter. In this case, the solution of the INEVP required 27 iterations.

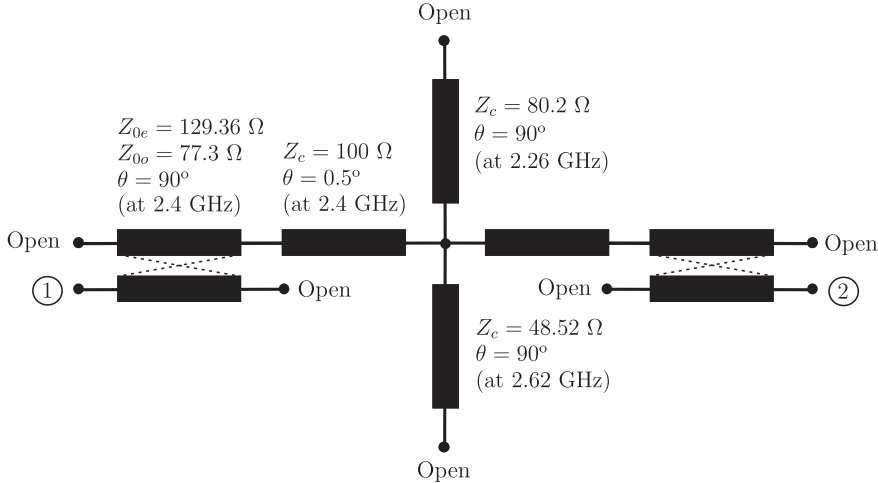

Fig. 11. Circuit schematic of the ideal transmission-line circuit realization of the third-order bandpass filter with one Type-II AFVC-topology in Fig. $5(d)$.

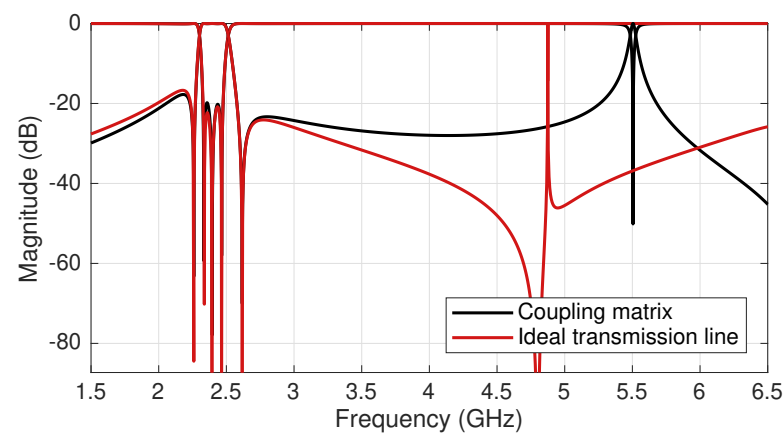

Fig. 12. Theoretical power transmission $\left(\left|S_{21}\right|\right)$ and reflection $\left(\left|S_{11}\right|\right)$ responses in a wide frequency interval of the transmission-line-based thirdorder bandpass filter with one Type-II AFVC along with those corresponding to its coupling-matrix synthesis-topology in Fig. 5(d).

\section{EXPERIMENTAL RESULTS}

For practical-validation purposes, one of the filter synthesis examples presented in Section $\mathrm{V}$ has been manufactured in microstrip technology and characterized. It corresponds to third-order bandpass filter design shaped by two resonators and one inter-resonator Type-II AFVC, which adds the two finite TZs and increases the filter order by one. The prefixed specifications are those that were previously indicated.

By using the coupling-matrix parameters given in Tables I and II, the filter was implemented as a distributed-element circuit network shaped by following elements: two quarterwavelength open-ended transmission lines corresponding to two series-type resonators (the additional $0.5^{\circ}$-long line segments are added to make them to exactly resonate at 2.387 $\mathrm{GHz}$ in accordance with the theoretical synthesis results), two open-ended stubs associated to the Type-II AFVC, and input and output couplings realized through parallel-coupled-line stages. The ideal transmission-line circuit schematic of the filter is depicted in Fig. 11. Its theoretical power transmission and reflection parameters, along with those corresponding to its associated coupling-matrix synthesis, are plotted in Fig. 12.

The layout - with indication of dimensions - and a photograph of the developed microstrip filter prototype are shown in Fig. 13. For circuit manufacturing, an Isola 680-338 substrate with the following parameters was used: relative dielectric 

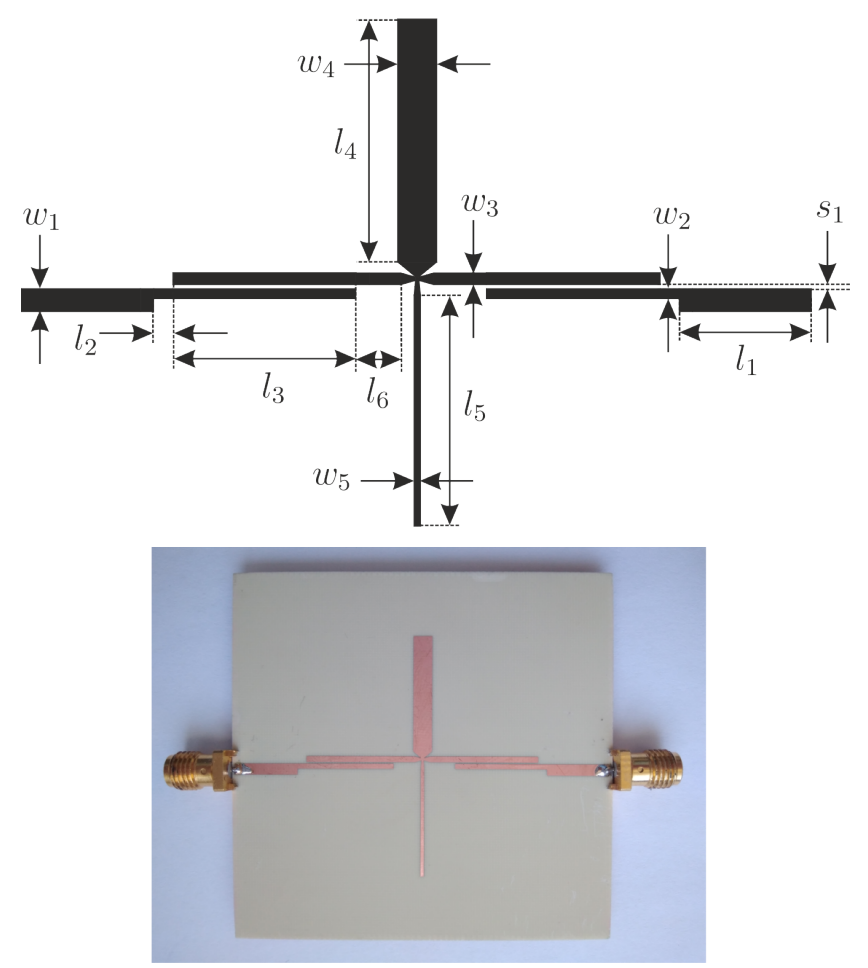

Fig. 13. Layout and photograph of the manufactured microstrip filter prototype (non-redundant dimensions in $\mathrm{mm}: w_{1}=1.76, w_{2}=0.78$ $w_{3}=0.93, w_{4}=2.98, w_{5}=0.51, l_{1}=10, l_{2}=1.42, l_{3}=13.85$, $l_{4}=18.38, l_{5}=17.43, l_{6}=3.37$, and $s_{1}=0.27$; the taper connections in the cross junction are $1-\mathrm{mm}$ long and terminate in $0.3-\mathrm{mm}$ width).

permittivity $\varepsilon_{r}=3.38$, dielectric thickness $H=0.762 \mathrm{~mm}$, metal thickness $t=17.5 \mu \mathrm{m}$, and dielectric loss tangent $\tan \left(\delta_{D}\right)=0.0035$. Although the obtained theoretical values for the different filter parameters were exploited as initial solution for the dimensional synthesis, their dimensions were finely adjusted with the zero-pole optimization technique available in the 3D finite-element-method electromagnetic software package InventSim ${ }^{\mathrm{TM}}$. The final simulations, taking into account the dielectric and conductor loss as well as the finite metal thickness, were carried out in ADS Momentum ${ }^{\mathrm{TM}}$. The measurements were performed with a PNA-X N5242A network analyzer from Agilent Technologies.

The simulated and measured power transmission and reflection parameters of the manufactured microstrip filter prototype are compared in Fig. 14. As can be seen, apart from some discrepancies in terms of a spectral shifting to a lowerfrequency range and some bandwidth reduction that are attributed to the tolerances of the manufacturing process and the relative dielectric permittivity, the filter principle is fairly verified. The main measured characteristics of the built filter prototype are as follows: center frequency of $2.377 \mathrm{GHz}, 3-$ $\mathrm{dB}$ absolute bandwidth equal to $130 \mathrm{MHz}-$ i.e., equal to $5.42 \%$ in relative terms-, minimum in-band power-insertionloss level of $1.18 \mathrm{~dB}$, minimum in-band power-matching level of $23.7 \mathrm{~dB}$, and TZs located at $2.238 \mathrm{GHz}$ and $2.584 \mathrm{GHz}$.

\section{CONCLUSION}

A new model for multi-coupled-resonator filters with networks comprising non-ideal AFVCs and NRNs was proposed.
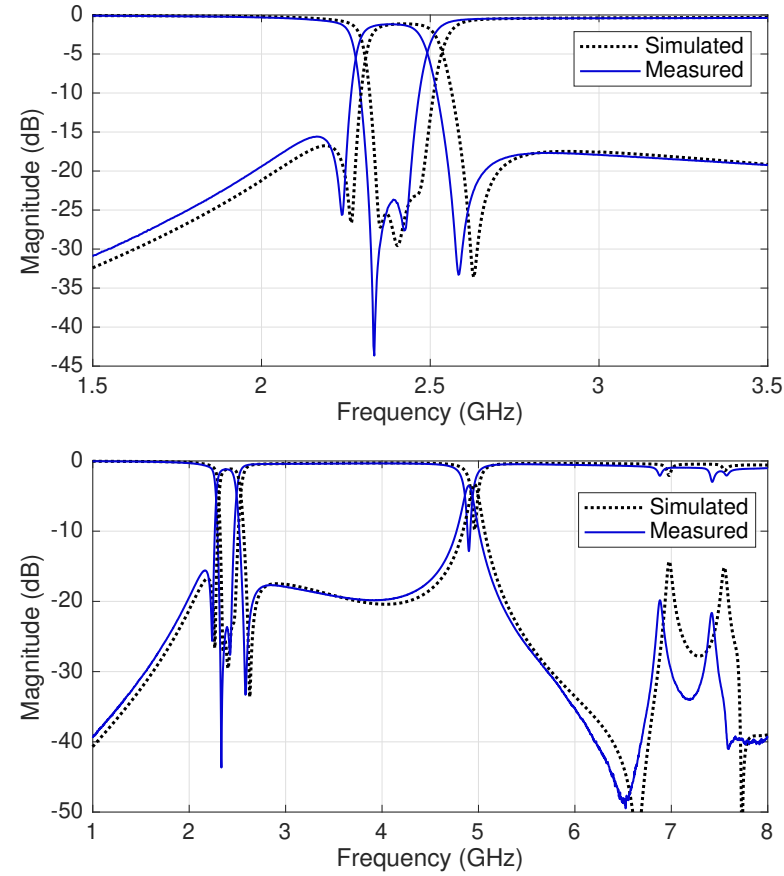

Fig. 14. Measured and simulated power transmission $\left(\left|S_{21}\right|\right)$ and reflection $\left(\left|S_{11}\right|\right)$ responses of the manufactured microstrip prototype corresponding to the third-order bandpass filter with one Type-II AFVC-topology in Fig. 5(d).

The synthesis procedure for determining the coupling matrix described by this general model was developed by using an inverse nonlinear eigenvalue framework. The synthesis process solves the associated INEVP by optimization and requires knowledge of the reference polynomials, whose roots can be matched to the characteristic frequencies of the filter parameters (i.e., zeros and poles). Its convergence requires that the solution is reachable for a pre-selected filter configuration and frequency-variation profiles of its coupling elements. The proposed model and synthesis technique are fully general, allowing for AFVCs, loaded or unloaded NRNs, non-resonating modes, frequency-dependent source-load coupling, multiple frequency-variant cross-couplings, or even multiple dispersive couplings to connect the source/load to the filter network. Further research work to be carried out is the extension of this approach to more-challenging filter topologies, such as multi-band filters, multi-port filtering devices (e.g., multiplexers or frequency channelizers), and filters with resistive elements (e.g., enhanced-in-band-amplitude-flatness lossy filters and reflectionless/absorptive filters).

\section{REFERENCES}

[1] A. Atia, A. Williams, and R. Newcomb, "Narrow-band multiple-coupled cavity synthesis," IEEE Trans. Circuits Syst., vol. CAS-21, no. 5, pp. 649-655, Sep. 1974.

[2] A. Atia and A. Williams, "Narrow-bandpass waveguide filters," IEEE Trans. Microw. Theory Techn., vol. 20, no. 4, pp. 258-265, Apr. 1972.

[3] R. J. Cameron, "General coupling matrix synthesis methods for Chebyshev filtering functions," IEEE Trans. Microw. Theory Techn., vol. 47, no. 4, pp. 433-442, Apr. 1999.

[4] - "Advanced coupling matrix synthesis techniques for microwave filters," IEEE Trans. Microw. Theory Techn., vol. 51, no. 1, pp. 1-10, Jan. 2003. 
[5] R. Cameron, C. Kudsia, and R. Mansour, Microwave Filters for Communication Systems. New York: Wiley, 2007.

[6] S. Amari, F. Seyfert, and M. Bekheit, "Theory of coupled resonator microwave bandpass filters of arbitrary bandwidth," IEEE Trans. Microw. Theory Techn., vol. 58, no. 8, pp. 2188-2203, Aug. 2010.

[7] W. Meng, H.-M. Lee, K. A. Zaki, and A. E. Atia, "Synthesis of wideband multicoupled resonators filters," IEEE Trans. Microw. Theory Techn. vol. 59, no. 3, pp. 593-603, Mar. 2011.

[8] S. Amari, M. Bekheit, and F. Seyfert, "Notes on bandpass filters whose inter-resonator coupling coefficients are linear functions of frequency," in Proc. 2008 IEEE MTT-S Int. Microw. Symp., 2008, pp. 1207-1210.

[9] U. Rosenberg, S. Amari, and F. Seyfert, "Pseudo-elliptic direct-coupled resonator filters based on transmission-zero-generating irises," in Proc. 2010 Eur. Microw. Conf., 2010, pp. 962-965.

[10] S. Amari and J. Bornemann, "Using frequency-dependent coupling to generate finite attenuation poles in direct-coupled resonator bandpass filters," IEEE Microw. Wireless Compon. Lett., vol. 9, no. 10, pp. 404 406, Oct. 1999.

[11] P. Kozakowski, A. Lamecki, M. Mongiardo, M. Mrozowski, and C. Tomassoni, "Computer-aided design of in-line resonator filters with multiple elliptical apertures," in Proc. 2004 IEEE MTT-S Int. Microw. Symp., 2004, pp. 611-614.

[12] R. Levy, "New cascaded trisections with resonant cross-couplings (ctr sections) applied to the design of optimal filters," in Proc. 2004 IEEE MTT-S Int. Microw. Symp., 2004, pp. 447-450.

[13] L. Szydlowski, A. Lamecki, and M. Mrozowski, "Coupled-resonator filters with frequency-dependent couplings: Coupling matrix synthesis," IEEE Microw. Wireless Compon. Lett., vol. 22, no. 6, pp. 312-314, Jun. 2012.

[14] _ - "A novel coupling matrix synthesis technique for generalized Chebyshev filters with resonant source-load connection," IEEE Trans. Microw. Theory Techn., vol. 61, no. 10, pp. 3568-3577, Oct. 2013.

[15] L. Szydlowski, N. Leszczynska, and M. Mrozowski, "Generalized Chebyshev bandpass filters with frequency-dependent couplings based on stubs," IEEE Trans. Microw. Theory Techn., vol. 61, no. 10, pp. 3601-3612, Oct. 2013.

[16] Y. He, G. Macchiarella, Z. Ma, L. Sun, and N. Yoshikawa, "Advanced direct synthesis approach for high selectivity in-line topology filters comprising $N-1$ adjacent frequency-variant couplings," IEEE Access, vol. 7, pp. 41 659-41668, 2019.

[17] Y. Zhang, F. Seyfert, S. Amari, M. Olivi, and K.-L. Wu, "General synthesis method for dispersively coupled resonator filters with cascaded topologies," IEEE Trans. Microw. Theory Techn., vol. 69, no. 2, pp. 1378-1393, Feb. 2021.

[18] V. Zemlyakov, M. Tyaglov, and V. Shevchenko, "Compact quasi-elliptic waveguide filters on contoured resonant diaphragms," Journal of Electromagnetic Waves and Applications, vol. 34, no. 2, pp. 224-234, 2020.

[19] M. Latif, G. Macchiarella, and F. Mukhtar, "A novel coupling structure for inline realization of cross-coupled rectangular waveguide filters," IEEE Access, vol. 8, pp. 107527-107 538, 2020.

[20] H. Shaman and J.-S. Hong, "A novel ultra-wideband (UWB) bandpass filter (BPF) with pairs of transmission zeroes," IEEE Micow. Wireless Compon. Lett., vol. 2, no. 17, pp. 121-123, Feb. 2007.

[21] M. Sánchez-Renedo, R. Gómez-García, and R. Loeches-Sánchez, "Microstrip filters with selectivity improvement using the new concept of signal-interference source/load coupling," in Proc. 2013 IEEE MTT-S Int. Microw. Symp., 2013, pp. 1-4.

[22] X.-L. Huang, L. Zhou, M. Völkel, A. Hagelauer, and J.-F. Mao, "Design of a novel quarter-mode substrate-integrated waveguide filter with multiple transmission zeros and higher mode suppressions," IEEE Trans. Microw. Theory Techn., vol. 66, no. 12, pp. 5573-5584, Dec. 2018.

[23] D. Miek, P. Boe, F. Kamrath, and M. Höft, "Techniques for the generation of multiple additional transmission zeros in H-plane waveguide filters," Int. J. Microw. Wireless Technol., vol. 12, no. 8, p. 723-732, Oct. 2020

[24] Z. Bai, J. Demmel, J. Dongarra, A. Ruhe, and H. van der Vorst Templates for the solution of algebraic eigenvalue problems: a practical guide. SIAM, 2000.

[25] J. F. Epperson, An introduction to numerical methods and analysis. John Wiley \& Sons, 2013.

[26] P. Kowalczyk, "Complex root finding algorithm based on delaunay triangulation," ACM Transactions on Mathematical Software (TOMS), vol. 41, no. 3, pp. 1-13, 2015.

[27] J. J. Michalski, "Complex border tracking algorithm for determining of complex zeros and poles and its applications," IEEE Trans. Microw. Theory Techn., vol. 66, no. 12, pp. 5383-5390, Dec. 2018.
[28] V. Mehrmann and H. Voss, "Nonlinear eigenvalue problems: A challenge for modern eigenvalue methods," GAMM-Mitteilungen, vol. 27, no. 2, pp. 121-152, 2004.

[29] W.-J. Beyn, "An integral method for solving nonlinear eigenvalue problems," Linear Algebra and its Applications, vol. 436, no. 10, pp. 3839-3863, 2012.

[30] L. Szydlowski, A. Lamecki, and M. Mrozowski, "Synthesis of coupledlossy resonator filters," IEEE Microw. Wireless Compon. Lett., vol. 20, no. 7, pp. 366-368, Jul. 2010.

[31] Y. He, G. Wang, L. Sun, and G. Rushingabigwi, "Generalised direct matrix synthesis approach for lossless filters," IET Microw. Antennas Propag., vol. 11, no. 2, pp. 158-164, Jan. 2017.

[32] P. Kozakowski, A. Lamecki, P. Sypek, and M. Mrozowski, "Eigenvalue approach to synthesis of prototype filters with source/load coupling," IEEE Microw. Wireless Compon. Lett., vol. 15, no. 2, pp. 98-100, Feb. 2005.

[33] S. Bastioli and R. V. Snyder, "Nonresonating modes do it better!: Exploiting additional modes in conjunction with operating modes to design better quality filters," IEEE Microw. Mag., vol. 22, no. 1, pp. 20-45, Jan. 2021.

[34] G. Macchiarella, "Generalized coupling coefficient for filters with nonresonant nodes," IEEE Microw. Wireless Compon. Lett., vol. 18, no. 12, pp. 773-775, Dec. 2008.

[35] R. Gómez-García, J.-M. Muñoz-Ferreras, and D. Psychogiou, "Fullyreconfigurable bandpass filter with static couplings and intrinsicswitching capabilities," in Proc. 2017 IEEE MTT-S Int. Microw. Symp., 2017, pp. 914-917. 